\title{
How OBGYNs can assist in the prenatal care and delivery of western lowland gorillas
}

\author{
Mary E. Beste ${ }^{1}$, Amber Armstrong ${ }^{1}$, Martha Weber ${ }^{2}$, James W. Cook ${ }^{1,3 *}$
}

\author{
${ }^{1}$ Department of Obstetrics and Gynecology, School of Medicine, University of South Carolina, Columbia, SC USA \\ ${ }^{2}$ Department of Veterinary Medicine, Riverbanks Zoo and Garden, Columbia, SC USA \\ ${ }^{3}$ Office of Curricular Affairs, University of South Carolina, School of Medicine Columbia, SC USA
}

Received: 12 November 2020

Accepted: 15 December 2020

\author{
*Correspondence: \\ Dr. James W. Cook, \\ E-mail: James.Cook@uscmed.sc.edu
}

Copyright: ( $)$ the author(s), publisher and licensee Medip Academy. This is an open-access article distributed under the terms of the Creative Commons Attribution Non-Commercial License, which permits unrestricted non-commercial use, distribution, and reproduction in any medium, provided the original work is properly cited.

\begin{abstract}
Prenatal care and perinatal planning are paramount for successful delivery outcomes in human pregnancies and has been shown to be equally as important with non-human primates. The authors describe two pregnancies and deliveries of a 12-year-old primigravid western lowland gorilla, Macy. Macy's first pregnancy resulted in a stillbirth and was complicated by breech positioning, while her second pregnancy resulted in a viable infant. This case report outlines the prenatal care Macy received in her pregnancies and highlights the importance of ultrasound for dating and fetal evaluation. The authors discuss options for breech positioning and considerations for cesarean section. This article showcases how obstetricians and veterinarians can implement human obstetrical recommendations with non-human primates to improve delivery outcomes.
\end{abstract}

Keywords: Gorilla, Prenatal, Breech

\section{INTRODUCTION}

Prenatal care can be influential in determining birth outcomes. In humans, inadequate care can lead to low birth weights, preterm labor and even fetal death. ${ }^{1}$ Since stringent antenatal care plans are beneficial for human pregnancy management, it is probable that prenatal care can affect outcomes with non-human primates, the critically endangered western lowland gorilla. This management includes monitoring diet, weight gain, medication regimens, behavioral changes and adequate dating through ultrasound and urine pregnancy testing.

In human obstetrics, preterm vaginal breech deliveries have shown to increase perinatal mortality, while a planned caesarean section has shown to reduce rates of neonatal death. ${ }^{3}$ There is little published data for breech deliveries in non-human primates. One major data point discovered in the analysis of all gorilla births in human care since 1980 was a stillbirth rate of $13 \% .^{2,3}$ This statistic raises the question, what indications should prompt cesarean delivery? There has been one publication of vacuum assisted vaginal delivery for arrest of second stage of labor, and one cesarean section performed for fetal distress and pre-eclampsia, but no protocols have been discussed for breech presentation. ${ }^{2,4}$ Furthermore, approximately $30 \%$ of gorilla infants in human care do not live past their first year of life, with $50 \%$ of deaths occurring before birth or within the first few days of life. ${ }^{2}$ Comparing discrepancies in prenatal care for two different pregnancies of the same gorilla with one delivery that ended in a viable infant and the other a fetal death, could shape the management of prenatal care for future non-human primate deliveries.

This case report will look further into prenatal care and if breech presentation should be an indication for cesarean delivery. With the dwindling population of lowland gorillas, one can understand the importance of facilitating the expansion of this species through prenatal and 
delivery protocols with breeding under the supervision of veterinary care.

The authors discuss how OB/GYNs can implement human obstetric protocols of prenatal and perinatal care to improve fetal and maternal outcomes. With veterinary and OB/GYN teams working together, there can be improved care for western lowland gorillas' pregnancies and could help sustain their population.

\section{CASE REPORT}

Macy, a primigravid western lowland gorilla from Riverbanks Zoo \& Garden, was closely followed by zoo medical personnel and OB/GYNs in Columbia, SC during two pregnancies and deliveries. Her first pregnancy at the age of 12 resulted in a stillbirth infant in 2017 and her second pregnancy at 13 years of age, resulted in a viable infant in 2018.

\section{First pregnancy}

Prior to conception, Macy learned multiple maternal behaviors including vaginal presentation, perineal presentation, nipple presentation/manipulation and abdominal ultrasounds via operant conditioning. Macy was also desensitized to rubbing alcohol and electric shavers on abdomen to allow for better ultrasound images.

In 2016, one month prior to stopping birth control, Macy was started on two prenatal vitamin gummies. Keepers monitored breeding activity and worked on training Macy to urinate in a collection bucket on cue. The goal was to obtain regular urine pregnancy tests to help estimate gestational age and due date of the infant. The average western lowland gorilla has a gestation period between 250 to 285 days. ${ }^{5}$ Urine samples could not be obtained between the last negative pregnancy test on September 8 , 2016 and the positive pregnancy result on October 14, 2016. Therefore, her due date was broadly estimated to be between mid-April and June 27th. Based on breeding activity, her due date was estimated to be May 8, 2017 June 13, 2017.

Throughout her first pregnancy, there were no medical complications. The keepers increased her diet by $20 \%$ which consisted of romaine lettuce, celery, bell pepper, and a mixed variety of vegetation. Weights were recorded weekly, with a total weight gain of $19 \mathrm{~kg}$. Along with the prenatal vitamin, she was started on 1.5 tsp of calcium carbonate once a day prior to conception. She also received Miralax (17 g) as needed throughout her pregnancy for managing constipation.

As part of her prenatal care, Macy received daily training sessions via protected contact to assess and visualize vitals and body condition. Macy received prenatal obstetrical ultrasounds starting three months after pregnancy was confirmed to monitor the growth and development of the fetus. Ultrasounds in March and April showed good fetal movement, reassuring views of head and spine, and cardiac activity despite limited visualization. The placenta was found to be in anterior, fundic position and blood flow to the placenta and infant's brain was appreciated. On May 3, 2017 there was suspected breech positioning, however the ultrasound views were poor. One week later, on May 10, 2017 (day 209 of gestation from positive urine test) breech positioning was confirmed. Macy went into labor on May 11, 2017. Her water broke at 6:38 AM. At 7:15 AM, before zoo staff could intervene, the infant's feet were observed. The infant was in a footling breech position. Macy delivered the baby at 8:17 AM. While laboring, she frequently changed positions, pulled on the baby's leg and umbilical cord, but did not appear to be in distress or discomfort. Once the baby was delivered, there was no fetal movement. Macy cleaned the infant immediately and positioned the infant in nursing position. Macy held and cared for infant for 6 days. During training sessions, keepers were able to touch the infant daily and ask for maternal behaviors. Throughout these postpartum days the troop members were attentive and protective toward Macy.

She had no significant postpartum complications, there was minimal spotting and she recovered appropriately. Macy began breeding again two weeks postpartum and remained off of birth control.

\section{Second pregnancy}

Macy continued prenatal vitamin gummies and 1.5tsp of calcium carbonate prior to conception. Keepers were able to obtain urine samples almost weekly. Her first recorded positive pregnancy test was on March 2, 2018. There were 15 days between the last negative test and the positive test on March 2nd. Thus, her estimated delivery date was between September 19, 2018-November 28, 2018. Macy did not receive a $20 \%$ increase in diet during her second pregnancy. Due to seasonal differences, more natural browse was available in her habitat and a diet increase was not needed to support weight gain. Keepers monitored her weight and behavior closely. Macy gained a total of $15 \mathrm{~kg}$ during her second pregnancy.

Prenatal ultrasounds began in June. Ultrasounds were performed every two weeks in June and July. Starting in August, ultrasounds were performed weekly. Heart rate was recorded to be $120-132$ bpm during multiple ultrasounds and the infant was noted to be cephalic during the ultrasounds leading up to birth. (Normal heart rate 120-180)1 On October 17, 2018, Macy was weighed in the morning, followed by her normal training routine. Behavior and appetite were normal. Troop was shifted onto exhibit per normal routine. Around 1:15 PM, Macy was observed being in labor and crowning on exhibit. At 1:17 PM, Macy successfully gave birth to a live infant with no complications, she was cleaning and licking the infant immediately after giving birth. Her pregnancy 
lasted 229 days from positive urine pregnancy test to delivery. There were no postpartum complications and Macy began rearing the infant appropriately.

\section{DISCUSSION}

The western lowland gorilla (Gorilla gorilla gorilla) is a critically endangered species that could benefit from the aid of the collaboration between OB/GYNs and veterinarians to improve breeding in human care. On average, there are $<50$ births per year of the lowland gorillas worldwide. ${ }^{6}$

Prenatal care can aid in monitoring a healthy pregnancy and is most beneficial implemented early to improve maternal and perinatal outcomes. ${ }^{7}$ It allows the mother and fetus to be frequently monitored, in order to troubleshoot and intervene appropriately if either gorilla develops serious health ailments. The surveillance of diet, growth, vitals, and behavior change are all beneficial to keep the mother and fetus healthy. Multiple human studies have discovered the importance of prenatal labs and screening tests to maintain maternal and fetal health. ${ }^{8}$ Even though pregnant gorillas due not undergo blood testing, the importance of temperature, growth and behavior change give substantial information to evaluate and treat conditions of pregnancy. Macy had an appropriate diet in both pregnancies and did not have trouble maintaining a healthy weight. In her second pregnancy, she did not receive a $20 \%$ increase in diet, but had appropriate weight gain in pregnancy. In humans, inadequate diets can lead to growth retardation. ${ }^{9}$ The different in diet and weight gain of the two pregnancies was a confounding issue in our case study. Macy adhered to a $20 \%$ increase in diet during her first pregnancy with a $19 \mathrm{~kg}$ weight gain. While in her second pregnancy she did not receive the increase in diet due to the time of year and increased vegetation in her habitat and gained $15 \mathrm{~kg}$. In this case, since the baby was shown on ultrasound growing appropriately up until delivery, it was most likely the breech presentation and 99 minutes the baby was entrapped in the birth canal that led to the demise. In addition, her medical management was adhered to consistently for both pregnancies with prenatal vitamins and calcium carbonate. Macy received daily training sessions via protective contact. Behavioral patterns and temperature gun measurements were recorded for any signs of distress, infection, or impending labor. Throughout both pregnancies Macy did not experience any complications and was relatively healthy.

Furthermore, the prenatal ultrasound screenings proved to be helpful with monitoring fetal growth and positioning. In humans, prenatal ultrasound is useful to determine growth restriction, dating, fetal location, fluid index, placenta previa and multiple gestations. ${ }^{10}$ In our case study, due to inconsistent urine pregnancy collection and the wide range for gorilla gestation (250-285 days), we were unable to know the correct delivery date of her first pregnancy. ${ }^{5}$ The ultrasounds conducted primarily looked at fetal heart rate, location of the fetus, fluid index and anatomy of the fetus. There has been data published measuring crown-rump length and biparietal diameter in marmosets that accurately predicted delivery dates within 5 days. $^{7}$ Furthermore, in the Journal of zoo and wildlife Medicine, the use of real time ultrasound with gorillas was shown to be useful in determining growth, primarily with serial measurements of the biparietal diameter. ${ }^{11}$ In Macy's pregnancies, the keepers and veterinary staff did not measure this due to limited views. These measurements could provide valuable information on gestational age, as data is acquired, and for future pregnancies this measurement is recommended. Ultrasound alone does not reduce maternal and fetal mortality but knowing the proper gestational age and detecting abnormalities early can alter treatment and improve outcomes. ${ }^{12}$ As a result, from the urine pregnancy tests, Macy's pregnancy was potentially a preterm birth. It is important to narrow down the gestational age, as a preterm delivery could be devastating. After recognizing the breech presentation, an emergency cesarean section protocol could have been planned for and put into place. With evaluating gorillas using the same protocol as humans, we can gather more comprehensive data to better treat this population.

No case studies have been published describing a breech delivery or the interventions available. In our case study, it was possible that the breech presentation was a major factor that led to the fetal demise. In humans, studies have shown that full term breech vaginal deliveries in comparison to cesarean section do not have increased maternal or infant morbidity or mortality; however, in preterm fetuses, there is significantly higher rate of neonatal mortality. ${ }^{12,13,14}$ With this information and the outcome of Macy's first pregnancy, breech presentation in a preterm gorilla fetus could be a relative contraindication to vaginal delivery in future pregnancies for western lowland gorillas. Another important consideration with vaginal delivery is the inability to monitor fetal heart tracings during labor, making it difficult to evaluate fetal status. Opting for a breech vaginal delivery can reduce the risk of many post cesarean section complications, including postpartum hemorrhage and uterine rupture. Additionally, the inability for the mother and her baby to bond immediately after birth is a concern when contemplating cesarean delivery and could affect the relationship of the mother and baby as well as the troop's dynamic. Coinciding, many western lowland gorillas do not have additional pregnancies after cesarean section due to the perceived risk of complications in future pregnancies. As a result, increased cesarean sections could affect numbers for breeding in veterinary care.

Additionally, an alternative for breech presentation used in human obstetrics is the external cephalic version. There have been seven successful external cephalic versions completed with baboons; however, with the body habitus of the western lowland gorilla, this could be 
a difficult procedure to perform. ${ }^{15}$ During the version, some baboons experienced bradycardia and vaginal bleeding during the procedure, but all made a full recovery. ${ }^{15}$ This intervention should be a consideration when assessing a breech presentation, and could be an option for future gorilla pregnancies.

In cases of emergency, a cesarean section is available. In the world of non-human primate births, cesarean section is not the initial option due to the high success of natural birthing and degree of postpartum complications with surgery. However, when the mother or fetus is under fatal distress, c-section has been documented to be a successful option. ${ }^{16}$ Taking the risks of postpartum complications and perinatal mortality in mind, each case should be evaluated thoroughly, and an emergency cesarean section should be considered early with a breech presentation.

\section{CONCLUSION}

A high mortality rate for captive born western lowland gorillas can impact the success of breeding programs, and the etiology of these poor obstetrical outcomes is not well understood. Our comparison allowed us to try and determine what might have contributed to a poor outcome. We conclude that Breech presentation may be a component to poor outcomes, and believe that Cesarean section may play a role, however we feel that risks and benefits of cesarean delivery need to be evaluated on a case-by-case basis. Further research should look at the rate of breech presentations in all Gorilla births, and what the outcomes of fetal lie has on neonatal death rates. Furthermore, more analysis of the effect of having had a prior Cesarean Section on a Gorilla's ability to safely reproduce would be of benefit. We also believe that research looking at the impact of dietary intake on pregnancy outcomes would provide useful data. Any and all case reports about the use of external cephalic version on western lowland gorillas would be welcomed.

\section{ACKNOWLEDGMENTS}

The authors would like to acknowledge the Veterinary staff and curators of the Riverbanks Zoo and garden for their dedication to the western lowland gorilla population and responsibility for the care of Macy, baby and all the other gorillas in their troop.

Funding: No funding sources Conflict of interest: None declared

Ethical approval: Not required

\section{REFERENCES}

1. Tardif S, Carville A, Elmore D, Williams LE, Rice K. Reproduction and Breeding of Nonhuman
Primates. Nonhuman Primat Biomedic Res. 2012:197-249.

2. Cole RE. Obstetric management of a protracted labor in a captive western lowland gorilla. Am J Obstet Gynecol. 2000;182(6):1306-11.

3. Sana S, Rebecca L, Fidino, Ross, Stephen. Stillbirth rates across three ape species in accredited American zoos. Am J Primatol. 2018;80:e22870.

4. Tayebi T, Zahrani ST, Mohammadpour R. Relationship between adequacy of prenatal care utilization index and pregnancy outcomes. Iran $\mathbf{J}$ Nurs Midwifery Res. 2013;18(5):360-6.

5. Harcourt AH, Stewart KJ. Gorilla society: conflict, compromise, and cooperation between the sexes. University of Chicago Press; 2008 Sep 15.

6. Mace GM. The genetic and demographic status of the Western lowland gorilla (Gorilla g. gorilla) in captivity. J Zool. 1988;216(4):629-54.

7. Smith A, Barr WB, Bassett-Novoa E, LeFevre N. Maternity Care Update: Prenatal Care and Specific Conditions. FP Essent. 2018;467:17-24.

8. Marangoni F, Cetin I, Verduci E, Canzone G, Giovannini M, Scollo P, et al. Maternal Diet and Nutrient Requirements in Pregnancy and Breastfeeding. An Italian Consensus Document. Nutrients. 2016;8(10):629.

9. Kind KL, Moore VM, Davies MJ. Diet around conception and during pregnancy--effects on fetal and neonatal outcomes. Reprod Biomed On. 2006;12(5):532-41.

10. Konje JC, de Chazal R, Taylor DJ. Routine ultrasound scanning in pregnancy. The benefits are clinical and psychological. Bio Med J. 1993;307(6903):559.

11. O'Grady JP. Practical Applications of Real Time Ultrasound Scanning to Problems of Zoo Veterinary Medicine. J Zoo Animal Med. 1978;9(2):52-6.

12. Jennewein L, Kielland-Kaisen U, Paul B, Möllmann CJ, Klemt AS, Schulze S, et al. Maternal and neonatal outcome after vaginal breech delivery at term of children weighing more or less than $3.8 \mathrm{~kg}$ : A FRABAT prospective cohort study. PLoS One. 2018;13(8):e0202760.

13. Gasim TG. Preterm singleton breech delivery in a teaching hospital of saudi arabia: vaginal versus cesarean delivery. J Fam Comm Med. 2008;15(2):65-70.

14. Nordin NM. An audit of singleton breech deliveries in a hospital with a high rate of vaginal delivery. The Malays J Medic Sci. 2007;14(1):28-37.

15. Abee, C, Mansfield K, Tardif, Suzette, Morris T. Nonhuman Primates in Biomedical Research. 2012.

16. Walter SL, Ali A, Killick R, Day C, Barrows M, Cahill DJ. Caesarean delivery of a Western Lowland gorilla with fetal distress and suspected preeclampsia. Int J Reprod Contracept Obstet Gynecol. 2018;7:2933-6.

Cite this article as: Beste ME, Armstrong A, Weber M, Cook JW. How OB/GYNS can assist in the prenatal care and delivery of western lowland gorillas. Int J Reprod Contracept Obstet Gynecol 2021;10:336-9. 